\title{
Evaluation of the Usefulness of the MODS Assay for the Detection of TB and MDR TB among PTB Suspects in the Philippines
}

\author{
Myrna T Mendoza ${ }^{1^{*}}$, Marc Agnew M Cajucom² ${ }^{2}$ Jill R Itable ${ }^{1,3}$ and Rosally P Zamora ${ }^{1,4}$ \\ ${ }^{1}$ Section of Infectious Diseases, Department of Medicine, University of the Philippines-Philippine General Hospital, Philippines \\ ${ }^{2}$ Microbiology Laboratory, Medical Research Laboratories, Department of Medicine, University of the Philippines-Philippine General Hospital, Philippines \\ ${ }^{3}$ Section of Infectious Diseases, Department of Internal Medicine, San Pedro Hospital, Davao City, Philippines \\ ${ }^{4}$ Department of Internal Medicine, East Avenue Medical Center, Quezon City, Philippines
}

"Corresponding author: Myrna T Mendoza, Section of Infectious Diseases, Department of Medicine, University of the Philippines-Philippine General Hospital, Philippines, Tel: +63-2-5251062; E-mail: myrnatmendoza@yahoo.com

Received date: January 30, 2015; Accepted date: August 07, 2015; Published date: August 13, 2015

Copyright: ( $) 2015$ Mendoza MT, et al. This is an open-access article distributed under the terms of the Creative Commons Attribution License, which permits unrestricted use, distribution, and reproduction in any medium, provided the original author and source are credited.

\section{Introduction}

In a developing country with high prevalence of pulmonary tuberculosis (PTB) like the Philippines, a reliable low cost diagnostic tool for the detection of Mycobacterium tuberculosis (M. tuberculosis) in clinical specimens is needed [1]. Case detection is mainly based on sputum microscopy, a diagnostic tool that has low sensitivity $[2,3]$. Mycobacterial cultures are more sensitive; however the standard egg based culture needs 6 weeks- 8 weeks of incubation before a result is released. The more rapid liquid based automated culture systems give results earlier than the standard methods but these are found only in big tertiary hospitals in our country and are generally considered to be expensive [4-6].

Moreover multi-drug resistant M. tuberculosis (MDR-TB) defined as resistance to Isoniazid $(\mathrm{H})$ and Rifampicin (R) and is a growing concern in the country. The 1997 National Tuberculosis Prevalence Survey reported an MDR rate of 4.3\% [7]. Methods available for drug susceptibility testing (DST) with solid media also takes time. On the average, DST results are released 3 weeks after a positive specimen is detected. M. tuberculosis culture and DST results therefore are known to the clinicians in about 3 months after a positive specimen is submitted. This is a long delay in the proper management of a patient with MDR-TB.

According to the World Health Organization global tuberculosis control update in 2013, of the 8.6 million incident cases of tuberculosis (TB), 450,000 (5\%) have MDR-TB, and there was an estimated 170,000 deaths from this disease [8]. Globally, only $48 \%$ of MDR-TB patients in the 2010 cohort of detected cases were successfully treated, reflecting high mortality rates and loss to follow-up. In addition, TB detection in general is still a problem. About $75 \%$ of the estimated 2.9 million missed TB cases were in 12 countries, including the Philippines [8].

The microscopic observation drug susceptibility (MODS) assay, a low cost rapid technique to detect $M$. tuberculosis from clinical specimens has been developed. This assay utilizes liquid culture media in plastic microwells where specimens are inoculated and microbial growth is observed through an inverted light microscope for the formation of the characteristic cording pattern of growth of $M$. tuberculosis [9]. This method easily permits $M$. tuberculosis detection and determination of drug susceptibility [10,11]. Positive results are usually observed within a week with an average detection time of 7 days-9 days [11]. The advantage of MODS for early detection of $M$. tuberculosis from clinical specimens has been evaluated in earlier studies in Peru and other countries where the burden of TB is high $[12,13]$. MODS have also been evaluated for early detection of drug resistant $M$. tuberculosis. With MODS, drug susceptibility tests with Isoniazid and Rifampicin were shown to be comparable with the standard LJ and agar based proportion method. Concordance rates reported were $99 \%-100 \%[13,14]$.

Recent publications of MODS studies show excellent results with M. tuberculosis detection with sensitivity and specificity of 73\%-89.1\% and $91 \%-99.1 \%$ respectively [15-17]. Comparing the sensitivity and specificity with recognized standards, MODS may turn out to be more accurate in isolating M. tuberculosis and predicting MDR-TB. Prompt detection of $M$. tuberculosis will result in a significant improvement in patient care management as well as implementation of infection control procedures.

In the Philippines, the development of a rapid, low cost and accurate diagnostic method in detecting tuberculosis in clinical specimens is urgently needed. With the limitations of various $\mathrm{TB} /$ MDR-TB diagnostic tests available in the country, there is a need to validate the usefulness of MODS, given its promising accuracy rates as reported from the literatures. This study is thus conducted to validate early detection of $M$. tuberculosis and MDR-TB using the MODS Assay in an actual bench setting in a TB diagnostic laboratory.

This study aims to compare the performance of the MODS assay in the detection of $M$. tuberculosis and MDR-TB from sputum specimens with the standard egg-based solid culture medium (Lowenstein Jensen) and the liquid MB BacT automated culture system. The specific objectives are to:

- determine time to detection of M. tuberculosis and MDR-TB from sputum specimen of patients with pulmonary tuberculosis using the MODS assay

- compare the accuracy rates of rifampicin (R) and isoniazid $(\mathrm{H})$ drug susceptibility test results of the MODS assay with the drug susceptibility tests results done with the agar-based disk proportion method

- determine the effect of on-going treatment on the isolation rate of M. tuberculosis by the MODS assay, standard egg based LJ medium and liquid based MB BacT automated culture system

- compare the cost of the advantage of the MODS assay with the standard egg-based medium (LJ), the liquid based automated MB $\mathrm{BacT}(\mathrm{MB})$ and the disk agar proportion method for detection of HR resistance. 
Page 2 of 6

\section{Method}

\section{Ethics}

The study protocol was approved by the Ethics Review Board of the University of the Philippines-Philippine General Hospital (UP-PGH) and the Research Institute of Tropical Medicine by the Department of Health (DOH).

\section{Study population and setting}

Sputum specimens were gathered from patients suspected to have pulmonary tuberculosis (PTB) were screened from two study populations: patients at the outpatient clinics of the departments of internal medicine, family medicine and TB DOTS clinic of the University of the Philippines-Philippine General Hospital (UP-PGH) and from patients referred by the Department of Health's Programmatic Management of Drug-Resistant tuberculosis (PMDT). PTB suspects were patients with signs and symptoms of chronic cough $>2$ weeks plus weight loss, with or without night sweats, unexplained fever, anorexia, chest pain, easy fatigability and body malaise. Patients referred by PMDT were mostly patients suspected to have multidrug resistant tuberculosis (MDRTB). MDR-TB suspects were patients who were for re-treatment and/or patients with past history of at least 1 month of treatment for PTB. Patients with on-going treatment were not excluded. These patients submitted 1-2 sputum specimens for $M$. tuberculosis culture and drug susceptibility tests.

All laboratory procedures were done at the UP-PGH TB Research Laboratory, a level II mycobacteriology laboratory. This laboratory was also identified as the culture center for PMDT by the DOH.

\section{Laboratory method}

We compared the result of the MODS Assay with the standard TB culture isolation procedures. This was conducted in parallel with the standard lowenstein jensen (LJ) culture method; MB BacT automated broth culture and the agar disk $1 \%$ proportion method for drug susceptibility testing. Only one medical technologist was specially trained to perform the MODS assay and he was blinded from the results of the culture performed using the LJ media, MB/BacT system and the results of the drug susceptibility proportion method. Another medical technologist interpreted the drug susceptibility testing using the agar disk $1 \%$ proportion method as described in CLSI and by Kent \& Kubica $[18,19]$

\section{Processing of sputum specimens}

Direct acid fast bacilli (AFB) microscopy smears were done using Ziehl-Neelsen staining method to all specimens. The sputum specimens were digested and decontaminated using the Petroffs Method [19]. The $4 \%$ sodium hydroxide is the reagent routinely used at the TB Research Laboratory in UP-PGH. An equal volume of sputum was added to $4 \%$ sodium hydroxide. The mixture is homogenized by vortex and then left to stand for 15 minutes, after which the tube was filled with phosphate buffer solution ( $\mathrm{pH}$ 6.8). This was then centrifuged at $3000 \mathrm{Xg}$ for 15 minutes. The supernatant was poured off and the sediment was resuspended with PBS. An aliquot was used for parallel cultures with LJ, MB BacT system and MODS assay.

\section{Culture technique for M. tuberculosis detection}

After decontamination, $100 \mu \mathrm{l}$ of the resuspended sediment was inoculated onto each two tubes of LJ medium. Each tube was properly labeled with the sample number and date of inoculation. These tubes were incubated at $37^{\circ} \mathrm{C}$ and examined after 48 hours and then weekly up to 8 weeks. For positive LJ cultures, each isolate was examined for cultural morphology, growth rate and pigmentation. In addition, niacin production and nitrite reduction (described by Kent and Kubica) $[19,20]$ were used for identification of M. tuberculosis.

The MB BacT culture bottle was inoculated with $500 \mu \mathrm{l}$ of resuspended sample and cultures were monitored by the automated culture detection machine for growth up to 42 days ( 6 weeks). A bottle of MB BacT contains Middlebrook 7H9 broth with nutrients and antibiotic supplements. All positive cultures from LJ and $\mathrm{MB}$ were processed for indirect drug susceptibility using the $1 \%$ proportion method to check for drug resistance.

\section{Drug susceptibility test for MDR-TB}

Indirect agar-based disk submerged $1 \%$ proportion method was done for all positive LJ cultures and MB Culture System [21]. This method used appropriate anti-tuberculosis drug disks submerged in Middlebrook 7H10 Agar (Difco) supplemented with OADC (Becton Dickinson) and glycerol. Critical drug concentrations used were as follows: $0.2 \mu \mathrm{g} / \mathrm{ml}$ for Isoniazid and $1 \mu \mathrm{g} / \mathrm{ml}$ for Rifampicin. Procedures and Interpretations were based on Clinical Laboratory Standards (CLSI) for Antimycobacterial Drug Susceptibility and as described by Kent and Kubica $[18,19]$.

\section{The MODS assay}

The MODS assay was performed according to the methods described by Caviedes and Moore [9,11]. Briefly, MODS medium was prepared using Middlebrook 7H9 broth base, Bacto Casitone, OADC Growth Supplement (oleic acid, albumin, dextrose and catalase) and PANTA Antibiotic Supplement (polymixin, amphotericin B, nalidixic acid, trimethoprim, azlocillin). The assay was prepared in a 48-well tissue culture plastic plates. Each run included a positive control of the standard M. tuberculosis H37Rv, a negative control of MODS medium and the test sample from the processed specimens. The positive control well contained $100 \mu \mathrm{l}$ of standard M. tuberculosis H37Rv and 900ul of MODS medium. The negative control well contained $1000 \mu \mathrm{l}$ of MODS medium. The test sample well contained $250 \mu \mathrm{l}$ of resuspended test sample and $750 \mu \mathrm{l}$ of MODS medium only. Plates were sealed with optical film (BioRad), covered with low evaporation lid and incubated at $37^{\circ} \mathrm{C}$ for up to 40 days. The culture plates were examined under an inverted light microscope at 40X magnification every day from day 3 to 15 and twice weekly afterwards up to 40 days of incubation. Positive cultures were identified by its characteristic cord formation. Bacterial and fungal contaminants were noted during and up to the end of the observation period. Contamination was compared with the parallel cultures in $\mathrm{MB}$ and $\mathrm{LJ}$ medium.

In the same 48-well tissue culture plastic plates described above, direct drug susceptibility testing was performed at the same time. Two wells contained Isoniazid and Rifampicin each. The drug concentrations used were as follows: isoniazid $(\mathrm{H}) 0.2 \mu \mathrm{g} / \mathrm{ml}$ and rifampicin (R) $1 \mu \mathrm{g} / \mathrm{ml}$, these are similar to the critical drug concentrations of the indirect agar disk proportion method. The standard $\mathrm{H} 37 \mathrm{Rv}$ positive control well served as the drug sensitive 
Citation: Mendoza MT, Cajucom MAM, Itable JR, Zamora RP (2015) Evaluation of the Usefulness of the MODS Assay for the Detection of TB and MDR TB among PTB Suspects in the Philippines. Clin Microbiol 4: 213. doi:10.4172/2327-5073.1000213

Page 3 of 6

control in each run. Rifampicin and isoniazid resistance were detected as growth or presence of cord formation in the drug containing wells.

\section{Data analysis}

M. tuberculosis detection rates for MODS, MB and LJ were summarized. We compared the time to a positive culture for LJ, MB and MODS, respectively, in samples positive in at least one method using the Wilcoxon test and presented using the Kaplan-Meier survival curves.

The accuracy of MODS for diagnosis of MDRTB was then assessed using the indirect disk agar proportion method as the gold standard reference test. Confidence intervals for accuracy measures (sensitivities, specificities, positive and negative predictive values) were calculated. All statistical analyses and graph were performed with Stata version 12 .

\section{Results}

We included a total of 795 sputum specimens collected from January 2012 to November 2013. There were 405 sputum samples from UP-PGH patients and 390 from PMDT patients. Out of the 795 sputum specimens, 770 were analyzed when the contaminated samples and samples with NTM were removed (Figure 1). Of the 770 specimens 269 were smear-negative and 501 were smear-positive for AFB. Table I shows the detection rates with the three culture techniques based on the smear results. Higher yield for $M$. tuberculosis was observed from smear positive specimens. Contamination rates were $2.6 \%$ for $\mathrm{LJ}$ and $1.1 \%$ for both MB and MODS.

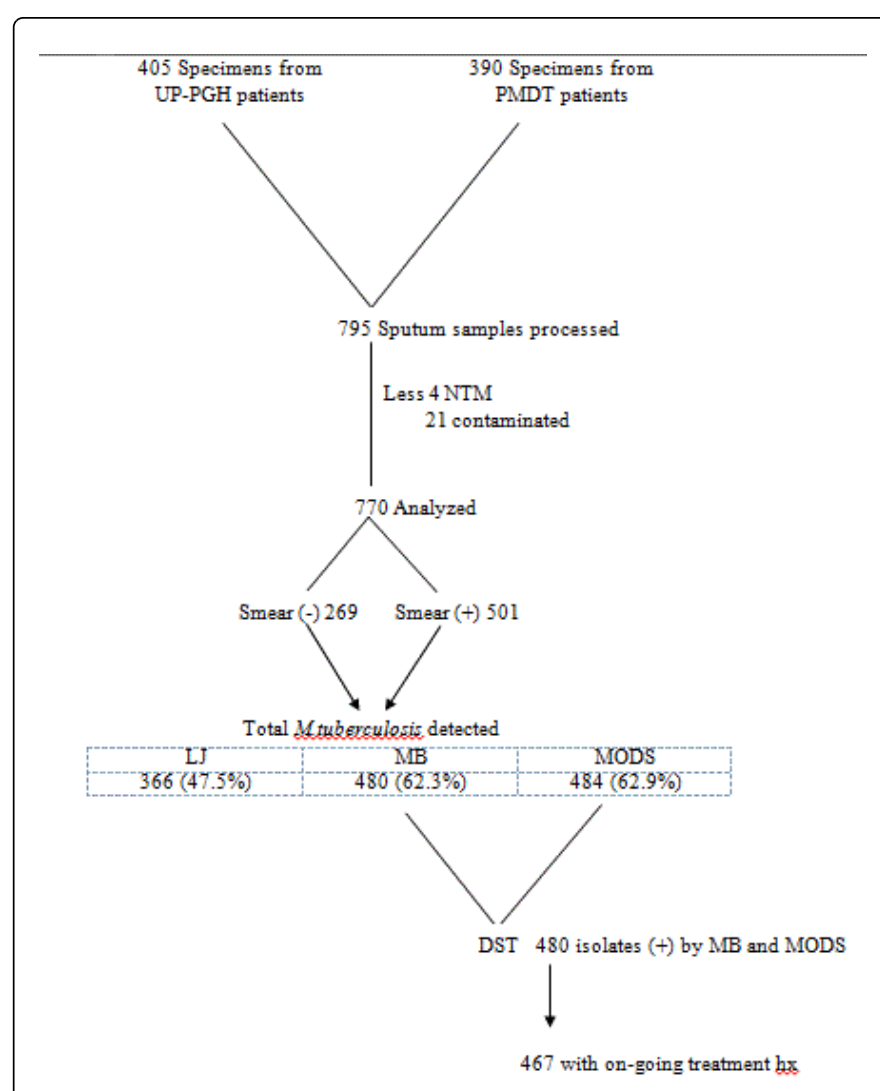

Figure 1: Methodology flow chart.

\begin{tabular}{|c|c|c|c|}
\hline Culture Technique & LJ & MB & MODS \\
\hline Smear-negative $(\mathrm{N}=269)$ & $33(12.3 \%)$ & $73(27.1 \%)$ & $77(28.6 \%)$ \\
\hline Smear-positive $(\mathrm{N}=501)$ & $333(66.5 \%)$ & $407(81.2 \%)$ & $407(81.2 \%)$ \\
\hline Total number of M. tuberculosis detected & $366(47.5 \%)$ & $480(62.3 \%)$ & $484(62.9 \%)$ \\
\hline NTM detected & 2 & 4 & 4 \\
\hline Contamination rate & $21(2.6 \%)$ & $9(1.1 \%)$ & $9(1.1 \%)$ \\
\hline
\end{tabular}

Table 1: Total number of $M$. tuberculosis detected in 770 specimens.

The rates of M. tuberculosis detection of the three techniques are shown in Figure 2. The median time for culture detection is 11 days for MODS, 17 days for MB and 37 days for LJ. The early detection of $M$. tuberculosis as shown in Figure 2 was statistically significant [Wilcoxon (Breslow) test], $p$ value $<0.0001$. Good agreement was obtained between LJ and MODS while excellent agreement was observed between MB and MODS (Kappa Statistic 0.73 and 0.99 for LJ vs MODS and MB vs MODS respectively $\mathrm{p}<0.0001$ ).

The average incubation period before culture detection was shorter in specimens with more bacillary load based on AFB smear grade. Compared with the negative smears, specimens with $1+$ and more had shorter incubation periods for positive MODS Assay ( $\mathrm{p}=0.000)$ (Table 2).

The rifampicin and isoniazid drug susceptibility test results using MODS and the standard disk proportion method are shown in Table
3. Computation of rates was based on 480 isolates that grew in both MB and MODS since both culture techniques utilized similar liquid culture media. There is better agreement of the isoniazid resistance rate compared with the standard disk proportion method.

The accuracy rates and predictive values of MODS in MDRTB detection are as follows: sensitivity $98.21 \%$ (CI 95, 99), specificity 99.60\% (CI 98, 99), PPV 99.56\% (CI 98, 100), NPV 98.41\% (CI 96, 100).

Out of the 480 culture positive specimens, 13 specimens had no source data on the history of treatment, 399 were from patients without history of treatment and 68 were from patients with on-going anti-TB treatment. M. tuberculosis was still isolated from sputum samples of patients with on-going anti-TB treatment and majority were multi-drug resistant (Table 4). 
Citation: Mendoza MT, Cajucom MAM, Itable JR, Zamora RP (2015) Evaluation of the Usefulness of the MODS Assay for the Detection of TB and MDR TB among PTB Suspects in the Philippines. Clin Microbiol 4: 213. doi:10.4172/2327-5073.1000213

Page 4 of 6

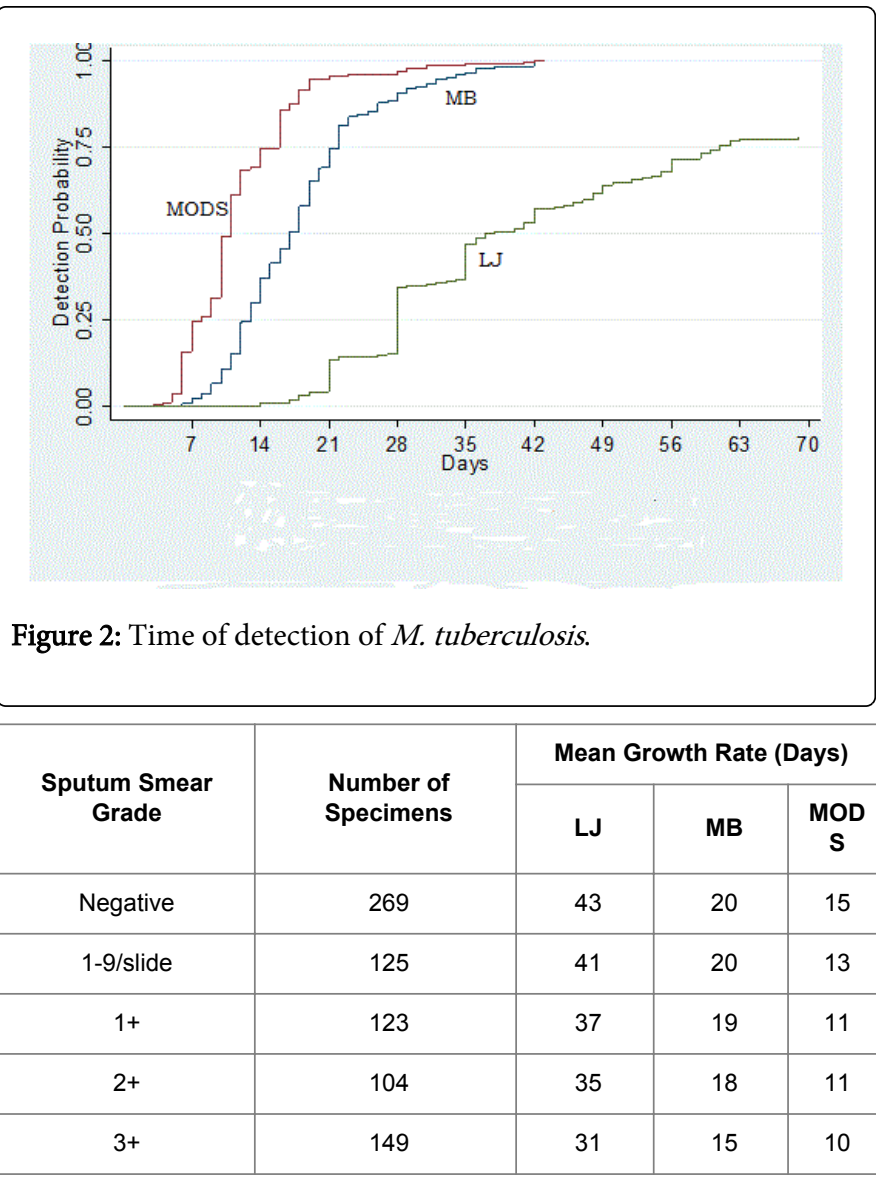

Table 2: Average incubation time based on smear grading $(\mathrm{N}=770)$.
The cost per sample tested using the three culture techniques were compared. Prices were based on the year 2009 cost of culture and drug susceptibility of $M$. tuberculosis at the UP-PGH laboratory. The automated culture system $(\mathrm{MB})$ is still the most expensive compared to LJ and MODS (Table 5). The cost of the added benefit of MODS over the standard LJ was computed as total cost of one test with MODS minus total cost with LJ divided by the \% difference in culture detection. The added cost benefit of MODS is only P5.70/test or approximately less than $\$ 1.00$.

\begin{tabular}{|c|c|c|}
\hline Resistance Pattern & \multicolumn{2}{|c|}{$\begin{array}{c}\text { Method of drug susceptibility test } \\
\text { (DST) }\end{array}$} \\
\hline & $\begin{array}{c}\text { Proportion Method } \\
\text { No. (\%) }\end{array}$ & $\begin{array}{c}\text { MODS No. } \\
\text { (\%) }\end{array}$ \\
\hline Susceptible to HR & $206(42.9)$ & $204(42.5)$ \\
\hline $\begin{array}{c}\text { Resistant to both Isoniazid and } \\
\text { Rifampicin (HR) }\end{array}$ & $231(48.1)$ & $228(47.5)$ \\
\hline Resistant to Rifampicin only (R) & $18(3.8)$ & $22(4.6)$ \\
\hline Resistant to Isoniazid only (H) & $25(5.2)$ & $26(5.4)$ \\
\hline
\end{tabular}

Table 3: Result of drug resistance from 480 M. tuberculosis isolates.

\section{Discussion}

Based on our findings, as early as first week of incubation, $25 \%$ of the culture positive specimens were detected by MODS assay. Also, by the second week of incubation, almost $90 \%$ of the positive specimens were detected. Mean growth rate even for smear negative specimens was 15 days. This is one week earlier than the MB and three weeks earlier than LJ. Instead of continuing incubation for 42 weeks, incubating for 30 days is enough to detect positive specimens for the MODS technique.

\begin{tabular}{|c|c|c|c|c|c|c|c|}
\hline \multirow{3}{*}{ Treatment Duration } & \multirow{3}{*}{$\begin{array}{c}\text { Total } \\
\text { Samples }\end{array}$} & \multicolumn{6}{|c|}{ Culture Technique } \\
\hline & & \multicolumn{2}{|c|}{ LJ } & \multicolumn{2}{|c|}{ MB } & \multicolumn{2}{|c|}{ MODS } \\
\hline & & No. $(+)$ & No. MDR & No. $(+)$ & No. MDR & No. $(+)$ & No. MDR \\
\hline No treatment history & 317 & 244 & 90 & 317 & 109 & 317 & 110 \\
\hline $1-2$ months & 108 & 80 & 64 & 108 & 86 & 108 & 82 \\
\hline 3-6 months & 15 & 9 & 5 & 15 & 10 & 15 & 10 \\
\hline$>6$ months & 13 & 10 & 8 & 13 & 11 & 13 & 11 \\
\hline Unknown duration & 14 & 13 & 8 & 14 & 8 & 14 & 8 \\
\hline No data & 13 & 10 & 6 & 13 & 7 & 13 & 7 \\
\hline Total & 480 & 366 & $181(49.4 \%)$ & 480 & $(48.1 \%)$ & 480 & $228(47.5 \%)$ \\
\hline
\end{tabular}

Table 4: Effect of on-going treatment on the isolation rate of $M$. tuberculosis $(\mathrm{N}=480)$.

\begin{tabular}{|c|c|c|c|}
\hline & LJ & MB & MODS \\
\hline Initial Outlay & & & \\
\hline MB Machine & - & $1,200,000$ & 349,000 \\
\hline Inverted Microscope & & & \\
\hline
\end{tabular}


Page 5 of 6

\begin{tabular}{|c|c|c|c|}
\hline \multicolumn{1}{|c|}{ Medical Technologist training } & & & 12,443 (10 days training) \\
\hline Average cost of culture media/reagents & 880 & 2200 & 960 (including HR powders for DST \\
\hline \multicolumn{1}{|l|}{ Average cost of drug susceptibility test } & - & $1,369.47$ & - \\
\hline Total cost (TC) for culture and DST & 880 & $3,569.47$ & 960 \\
\hline $\begin{array}{l}\text { Computation for added cost of benefit of MODS=P5.70 } \\
\text { [TC (MODS)-TCLJ/\%difference in yield] }\end{array}$
\end{tabular}

Table 5: Cost comparison market price of the three culture techniques in Philippine peso.

M. tuberculosis detection rate in our study was lower compared with the previous MODS evaluation studies because we did not exclude patients with on-going treatment. In a single-center study with high HIV prevalence by Shiferaw, their detection rate was $94 \%$ because they excluded patients on treatment [14]. Among non HIV patients, Minh $\mathrm{Ha}$ on the other hand excluded patients on treatment and their detection rate was only $50.5 \%$ [20]. Compared to a multi-center study in Brazil, we also included sputum samples of patients with on-going anti TB treatment [13]. Our overall detection rate was better $(62.9 \%$ vs 44.4\%).

Despite on-going treatment, $M$. tuberculosis was isolated in all culture techniques, but $56 \%-85 \%$ of the isolates were MDR. Both MB and MODS had similar detection rates for MDR. Even among those without treatment, the proportion of MDR were also high (43\%-46\%) and we attribute this to population bias since $49 \%$ of the sputum specimens came from MDRTB suspects. The early detection of MDR TB by MODS has important public health implications in terms of early case detection and management. During TB treatment, the usual intensive phase of HRZE for two months is reduced to HR for the four month maintenance period, without the knowledge of the DST [1]. Ideally the DST result should be known before drug regimen is reduced to HR especially for MDRTB suspects. Before shifting to maintenance treatment, MODS assay can provide this timely DST result to guide clinicians in the management of patients with MDRTB.

With the MODS assay, culture detection and HR resistance rate results are concomitantly available at the same time. In comparison to previous studies which showed sensitivity of $77.8 \%$-92\%our study provided better sensitivity results $[17,20,21]$. We attribute this to the concentration of isoniazid used for the MODS assay in this study. Isoniazid concentration was decreased to be similar to the critical concentration used for standard agar disk proportion method e.g. 0.2 $\mu \mathrm{g} / \mathrm{ml}$. In the prospective evaluation of MODS assay in Thailand, the isoniazid concentration used was $0.1 \mu \mathrm{g} / \mathrm{ml}$ when they compared MODS DST to MGIT 960. The MODS sensitivity and specificity for MDRTB in that study were $85.7 \%$ and $97.5 \%$ respectively [21].

We compared the cost of using the three techniques. All costs included media, reagent cost, medical technologist time, machine depreciation and $20 \%$ mark up for overhead cost which reflect the prices in 2009 when the protocol was made. LJ was the cheapest for MTB culture. With the MODS assay, for an additional cost of P5.70, the patient and clinician can receive the result 2 weeks earlier than LJ for the M. tuberculosis detection. And instead of waiting for an additional 3 to 4 weeks for the drug susceptibility test result, MODS can provide MDR test results at the same time as the culture result. Appropriate treatment can therefore be provided early to the patient especially for the MDRTB suspect.

\section{Limitation}

Our study is limited to clinical specimens. We did not take into account the condition of the patients especially those on treatment. The skill of the operator outside of the research laboratory could be a potential limitation of MODS due to the proficiency in discriminating the characteristic cording of M. tuberculosis from NTM. This however can be remedied with proper training. There are reports on MODS training of laboratory staff outside of research laboratories and results are encouraging [22,23]. Furthermore, other potential challenges include medical technologist time requirement and the need for detailed attention to laboratory procedures to prevent contamination. Standardization of the technique and quality control procedures should be addressed.

\section{Conclusion and Recommendations}

The MODS Assay is a rapid and cost-effective way of detecting $M$. tuberculosis comparable with $\mathrm{MB}$ automated culture system which also utilizes the liquid medium Middlebrook 7H9. We recommend the use of Isoniazid concentration of $0.2 \mu \mathrm{g} / \mathrm{ml}$ for the assay as this is similar to the critical drug concentration being used in the agar disk proportion method for DST.

With adequate training of medical technologists, the MODS Assay can be adopted in the regional TB laboratory centers in our country. This will actually lessen the waiting time for the results in a cheaper way. Since MODS can be applied to direct samples, this will give the clinician the edge of knowing the culture and sensitivity results earlier. MODS is cost beneficial to the patients.

\section{Acknowledgement}

This study was funded by the Philippine Council for Health Research and Development (PCHRD). The training of Mr. Marc Cajucom on MODS Assay was done at the Oxford University, Ho Chi Minh, Vietnam under the supervision of Dr. Maxine Caws. Statistical analysis was performed by Prof Maria Lourdes Amarillo.

\section{References}

1. Philippine Practical Guidelines Group in Infectious Diseases (PPGG-ID) (2006) The Philippine Clinical Practice Guidelines on the Diagnosis, Treatment, Prevention and Control of Tuberculosis 2: 6-7.

2. (2000) Diagnostic Standards and Classification of Tuberculosis in Adults and Children. This official statement of the American Thoracic Society and the Centers for Disease Control and Prevention was adopted by the ATS Board of Directors, July 1999. This statement was endorsed by the Council of the Infectious Disease Society of America, September 1999. Am J Respir Crit Care Med 161: 1376-1395. 
Citation: Mendoza MT, Cajucom MAM, Itable JR, Zamora RP (2015) Evaluation of the Usefulness of the MODS Assay for the Detection of TB and MDR TB among PTB Suspects in the Philippines. Clin Microbiol 4: 213. doi:10.4172/2327-5073.1000213

Page 6 of 6

3. Kothadia SN, Deshmukh S, Saoji AM (1990) Evaluation of direct microscopy as a screening test in the diagnosis of pulmonary tuberculosis Indian J Pathol Microbiol 33: 68-73.

4. Brunello F, Favari F, Fontana R (1999) Comparison of the MB/BacT and BACTEC $460 \mathrm{~TB}$ systems for recovery of mycobacteria from various clinical specimens. J Clin Microbiol 37: 1206-1209.

5. Mendoza MT, Reyes LF, Pascual M, Tan-Torres T (1993) Culture isolation of Mycobacterium tuberculosis Using the Radiometric Bactec System Compared with Conventional Lowenstein Jensen Medium. Phil J Microbiol Infect Dis 22: 17-22.

6. Ang CF, Mendoza MT, Bulatao WC, Cajucom MAM (2001) Culture Isolation of Mycobacteria by MB/BacT System Compared to Lowenstein Jensen Egg Medium Culture Method. Phil J Microbiol Infect Dis 30: 40-43.

7. Tupasi TE, Radhakrishna S, Rivera AB, Pascual ML, Quelapio MI, et al. (1999) The 1997 Nationwide Tuberculosis Prevalence Survey in the Philippines. Int J Tuberc Lung Dis 3: 471-477.

8. World Health Organization (2013) Global Tuberculosis Report 2013.

9. Caviedes L, Lee TS, Gilman RH, Sheen P, Spellman E, et al. (2000) Rapid Efficient Detection of Mycobacterium tuberculosis in Sputum by Microscopic Observation of Broth Cultures. J Clin Microbiol 38: 1203-1208.

10. Park WG, Bishai WR, Chaisson RE, Dorman SE (2002) Performance of the Microscopic Observation Drug Susceptibility Assay in Drug Susceptibility Testing for Mycobacterium tuberculosis. J Clin Microbiol 40: 4750-4752.

11. Moore DAJ, Mendoza D, Gilman RH, Evans CAW, Delgado MGH, et al. (2004) The Tuberculosis Working Group in Peru. Microscopic Observation Drug Susceptibility Assay, a Rapid, Reliable Diagnostic Test for Multidrug-Resistant Tuberculosis Suitable for Use in Resource-Poor Setting. J Clin Microbiol 42: 4432-4437.

12. Moore DA, Evans CA, Gilman RH, Caviedes L, Coronel J, et al. (2006) Microscopic-observation drug-susceptibility assay for the diagnosis of TB. N Engl J Med 355: 1539-1550.

13. Arias M, Mello FCQ, Pavon A, Marsico AG, Alvarado-Galvez C, et al. (2007) Clinical Evaluation of the Microscopic-Observation DrugSusceptibility Assay for Detection of Tuberculosis. Clin Infect Dis 44: 674-680.
14. Shiferaw G, Woldeamanuel Y, Gebeyehu M, Girmachew F, Demessie D, et al. (2007) Evaluation of microscopic observation drug susceptibility assay for detection of multidrug-resistant Mycobacterium tuberculosis. J Clin Microbiol 45: 1093-1097.

15. Makamure B, Mhaka J, Makumbirofa S, Mutetwa R, Mupfumi L, et al. (2013) Microscopic-observation drug-susceptibility assay for the diagnosis of drug-resistant tuberculosis in Harare, Zimbabwe. PLoS One 8: e55872.

16. Solomon S, Balakrishnan P, Vignesh R, Waldrop G, Solomon SS, et al. (2013) A rapid and low-cost microscopic observation drug susceptibility assay for detecting TB and MDR-TB among individuals infected by HIV in South India. Indian J Med Microbiol 31: 130-137.

17. Walusimbi S, Bwanga F, De Costa A, Haile M, Joloba M, et al. (2013) Meta-analysis to compare the accuracy of GeneXpert, MODS and the WHO 2007 algorithm for diagnosis of smear-negative pulmonary tuberculosis. BMC Infect Dis 13: 507.

18. Clinical Laboratory Standard Institute (2008) Laboratory Detection and Identification of Mycobacteria; Approved Gluideline.

19. Abate G, Aseffa A, Selassie A, Goshu S, Fekade B, et al. (2004) Direct colorimetric assay for rapid detection of rifampin-resistant Mycobacterium tuberculosis. J Clin Microbiol 42: 871-873.

20. Dang TM, Nguyen TN, Wolbers M, Vo SK, Hoang TT, et al. (2012) Evaluation of microscopic observation drug susceptibility assay for diagnosis of multidrug-resistant tuberculosis in Viet Nam. BMC Infect Dis 12: 49 .

21. Chaiyasirinroje B, Aung MN, Moolphate S, Kasetjaroen Y, Rienthong S, et al. (2012) Prospective evaluation of simply modified MODS assay: an effective tool for TB diagnosis and detection of MDR-TB. Infect Drug Resist 5: 79-86.

22. Mendoza A, Castillo E, Gamarra N, Huamán T, Perea M, et al. (2011) Reliability of the MODS assay decentralisation process in three health regions in Peru. Int J Tuberc Lung Dis 15: 217-22, i.

23. Elinav H, Kalter HD, Caviedes L, Moulton LH, Lemma E, et al. (2012) Training laboratory technicians from the Ethiopian periphery in the MODS technique enables rapid and low-cost diagnosis of Mycobacterium tuberculosis infection. Am J Trop Med Hyg 86: 683-689. 